\title{
Explanation of differences in experimental and computational results for the preferential concentration of inertial particles
}

\author{
Thorsten Wittemeier ${ }^{\mathrm{a}, *}$, John S. Shrimpton ${ }^{\mathrm{b}}$, \\ Faculty of Engineering and the Environment, University of Southampton, Southampton \\ SO17 1BJ, United Kingdom \\ ${ }^{a}$ T.Wittemeier@soton.ac.uk \\ ${ }^{b}$ John.Shrimpton@soton.ac.uk
}

\begin{abstract}
Using one-way coupled direct numerical simulations of inertial particles in homogeneous isotropic turbulence we investigate the non-linear dependence of local particle concentrations in particle clusters on the number density of particles used in the simulation per volume. We show that the Reynolds-number dependence of local concentrations in clusters can be predicted for sufficiently high Reynolds numbers by a scaling relation that depends on the average particle number density and the Kolmogorov length. Depending on the average particle number density and gravity, a maximum of preferential concentration does not necessarily occur at Stokes numbers around unity. This explains the differences observed between recent experimental and computational results.
\end{abstract}

Keywords: Turbulence, inertial particles, preferential concentration

\section{Introduction}

We present results of our investigations of the preferential concentration [1] of inertial particles in homogeneous isotropic turbulence using a pseudospectral direct numerical simulation (DNS) code.

Clustering of particles has been found over a wide range of Kolmogorov-scale

${ }^{*}$ Corresponding author 
Stokes numbers from $S t_{\eta}=0.05$ [2] up to several hundred [1]. Many workers have observed the strongest clustering around $S t_{\eta}=1$ using both measurements and DNS 2, 3, 4, 5]. In wind-tunnel experiments at Taylor-scale Reynolds numbers $R e_{\lambda}$ between 230 and 400 and $S t_{\eta}$ between 2 and 10, Obligado et al. 6] found peak preferential concentration at $S t_{\eta} \approx 4$. Sumbekova et al. [7] performed wind-tunnel experiments between $S t_{\eta}=0.1$ and $S t_{\eta}=5$ with $R e_{\lambda}$ between 170 and 500. They found no significant Stokes number dependence, but a strong dependence on both Reynolds number and volume fraction.

Using DNS between $R e_{\lambda}=65$ and $R e_{\lambda}=152$, Collins and Keswani [8] found that the radial distribution function $g(r)[9]$ seems to reach a plateau with increasing $R e_{\lambda}$. The results were confirmed by Ireland et al. 2] at $R e_{\lambda}$ up to $\sim 600$. However, quantities based on particle concentration, such as $D[3]$, exhibit a more complex behaviour. Peak $D$ has been observed to decrease with increasing Reynolds number [10].

Monchaux et al. 11] found that the particle concentration inside clusters had a non-linear dependence on the global particle loading. Sumbekova et al. [7] also observed a nonlinear increase of local particle concentrations inside clusters with the particle volume fraction in experiments up to $R e_{\lambda}=450$.

Aliseda et al. [5] found a maximum of $D$ at bin sizes corresponding to $10 \eta$ 25 (where $\eta$ is the Kolmogorov length) and concluded that $10 \eta$ is a typical average cluster size. Wood et al. [4] observed a peak at bin sizes between $8 \eta$ and $20 \eta$, with the peak shifting towards larger sizes with increasing $S t_{\eta}$. Sumbekova et al. 7] observed that average cluster dimensions increased with $R e_{\lambda}$, up to a size of about $100 \eta$ at $R e_{\lambda} \approx 500$.

Whereas the effects of Reynolds number, Stokes number and gravity on particle dynamics and particle-particle distances have been investigated numerically [2, 12], a systematic study of the parameters influencing cluster sizes and local particle concentrations in clusters has to our knowledge only been performed experimentally [7]. Our goal is to add to the quantitative understanding of particle clusters by investigating the influence of Reynolds number, Stokes number, average particle number density and gravity on $D[3]$, a measure that gives 


\begin{tabular}{|c|c|c|c|c|c|}
\hline Number of grid points & $N^{3}$ & {$[-]$} & $64^{3}$ & $512^{3}$ & $1024^{3}$ \\
\hline Resolution & $k_{\max } \eta$ & {$[-]$} & 1.5 & 1.1 & 1.0 \\
\hline Reynolds number & $R e_{\lambda}$ & {$[-]$} & 45 & 250 & 470 \\
\hline Fluid viscosity & $\nu$ & {$\left[\mathrm{m}^{2} / \mathrm{s}\right]$} & $2.5 \cdot 10^{-2}$ & $2.5 \cdot 10^{-4}$ & $9.9 \cdot 10^{-5}$ \\
\hline Kolmogorov length scale & $\eta$ & {$[\mathrm{m}]$} & $4.0 \cdot 10^{-2}$ & $3.7 \cdot 10^{-3}$ & $1.7 \cdot 10^{-3}$ \\
\hline Taylor length scale & $\lambda$ & {$[\mathrm{m}]$} & $5.2 \cdot 10^{-1}$ & $1.2 \cdot 10^{-1}$ & $7.4 \cdot 10^{-2}$ \\
\hline Integral length scale & $L$ & {$[\mathrm{~m}]$} & 1.0 & $8.0 \cdot 10^{-1}$ & $8.3 \cdot 10^{-1}$ \\
\hline Dissipation rate & $\epsilon$ & {$\left[\mathrm{m}^{2} / \mathrm{s}^{3}\right]$} & 6.3 & $8.0 \cdot 10^{-2}$ & $1.1 \cdot 10^{-1}$ \\
\hline Particle diameter & $d$ & {$[\mathrm{~m}]$} & $4.0 \cdot 10^{-3}$ & $3.7 \cdot 10^{-4}$ & $8.8 \cdot 10^{-5}$ \\
\hline Gravity & $g$ & {$\left[\mathrm{~m} / \mathrm{s}^{2}\right]$} & -9.81 & - & - \\
\hline
\end{tabular}

Table 1: Input parameters and fluid statistics of the simulations

quantitative information about the effect of preferential concentration on both local concentrations and cluster sizes. We use one-way coupled DNS, which makes the systematic variation of these parameters straightforward compared 40 to an experimental setup.

\section{Methods}

PANDORA [13] is a pseudospectral DNS code that is parallelised in 1-D (slab decomposition) and contains a Lagrangian point-particle implementation. The inertia, drag and gravity terms of the Maxey-Riley equation 14] are solved.

45 Fast Fourier transforms are performed using the library FFTW [15]. The forcing scheme is based on the stochastic scheme developed by Eswaran and Pope [16].

One-way coupled simulations of homogeneous isotropic turbulence have been performed on various grid sizes. The velocity field was initialised to a spectrum as described by Sullivan et al. [17]. The resolution of all model runs was in the order of $k_{\max } \eta \approx 1$ where $k_{\max }$ is the highest resolved wave number. Particles were introduced once the turbulence was considered statistically stationary, i.e. when the principle turbulent statistics were fluctuating around a mean over about 10 to 20 eddy turnover times rather than following a trend. The cartesian 
coordinates of the initial particle position were determined using the intrinsic

${ }_{55}$ Fortran random number generator. By interpolating the fluid velocity at the initial particle position the relative particle velocity was set to zero. In all simulations the Kolmogorov-scale Stokes number $S t_{\eta}$ was modified by changing the density $\rho_{p}$ of the particles while keeping the particle diameter fixed. A summary of input parameters and fluid statistics of the simulations is given

60 in table [1. We used the equilibrium dissipation law $\epsilon=C_{\epsilon} U^{3} / L$ [8], which relates dissipation rate $\epsilon$, turbulent kinetic energy $U^{2}$ and integral length scale $L$, to compare our DNS against a series of published results [19, 20, 21]. Our results are in good agreement with those of other workers, as shown in figure [1. To quantify preferential concentration, we used the $D$ measure [3] and the 65 Radial Distribution Function (RDF) [9]. D [3] compares the standard deviation of a measured particle distribution to the standard deviation of a randomly positioned set of particles by binning the particles in boxes of side length $h$. $D=0$ when the particle distribution is a Poisson distribution and $D<0$ for a uniform particle distribution [3]. The radial distribution function $g(r)$ [9] 70 represents the number of particle pairs at a given separation compared to a uniform particle distribution [22]. We use the Kolmogorov-scale Stokes number $S t_{\eta}=\frac{\tau_{p}}{\tau_{\eta}}[1]$, defined as the ratio of the particle relaxation time $\tau_{p}$ and the Kolmogorov time scale $\tau_{\eta}$.

\section{Results}

The results of simulations at $R e_{\lambda}=250$ for both $D$ and $g(r)$ at $S t_{\eta}=$ 0.57 and $S t_{\eta}=1.06$, in which the particle number density was varied between $N_{p}=503.9$ particles $/ \mathrm{m}^{3}$ and $N_{p}=40314.4$ particles $/ \mathrm{m}^{3}$, are shown in figure 2. As can be seen from the figure, the radial distribution function $g(r)$ shows no systematic dependence on the average particle number density (see (a) and (c)), whereas it has a strong effect on $D$ (see (b) and (d)). The maximum of $D$ increases with the number of particles, while its peak shifts towards smaller bin sizes. 


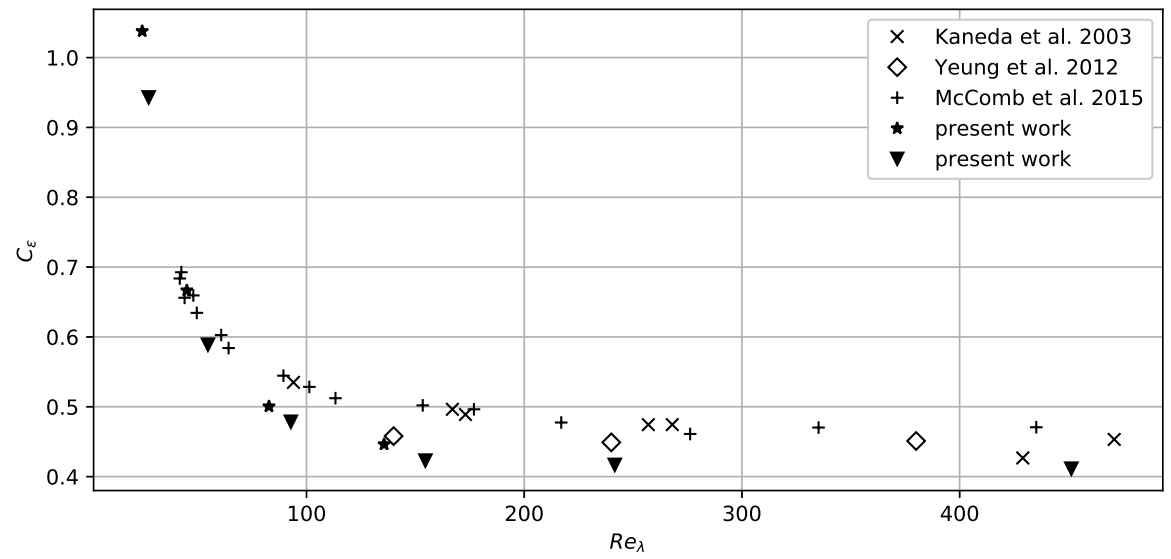

Figure 1: Comparison of the dissipation constant $C_{\epsilon}$ between our simulations and published results [19, 20, 21

(a) $S t_{\eta}=0.57$

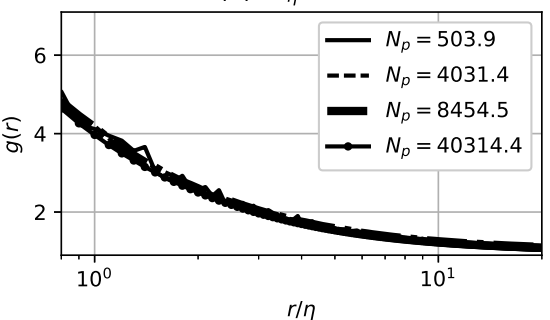

(c) $S t_{\eta}=1.06$

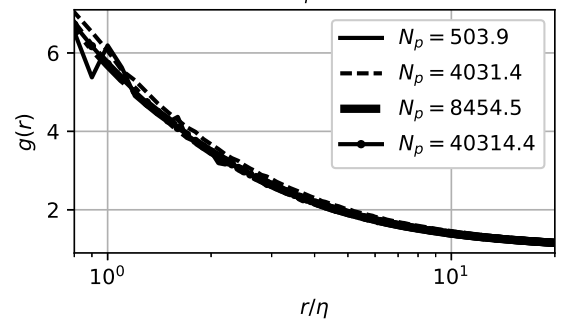

(b) $S t_{\eta}=0.57$

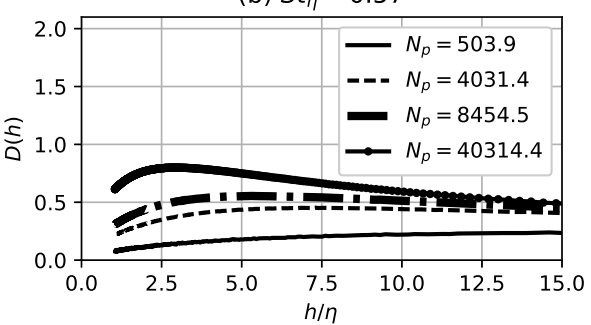

(d) $S t_{\eta}=1.06$

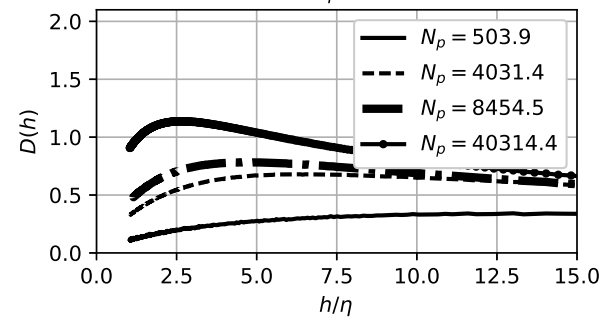

Figure 2: Influence of the average particle number density on the radial distribution function $g(r)$, left column, and the $D$ measure, right column, for $S t_{\eta}=0.57$ (upper row) and $S t_{\eta}=1.06$ (lower row). Simulations at $R e_{\lambda}=250$. 
As a compromise between computing time and a broad range of parameters, simulations at $R e_{\lambda}=45$ were performed for $S t_{\eta}$ between 0.4 and 8 , using between $N_{p}=16.5$ particles $/ \mathrm{m}^{3}$ and $N_{p}=8454.5$ particles $/ \mathrm{m}^{3}$. The results are shown in the left column of figure 3, $D$ is highest at $S t_{\eta}=1$. The shift of the peak position is stronger for more inertial particles and almost not visible for $S t_{\eta}<1$ particles, whereas the change in the maximum of $D$ is greater for low $S t_{\eta}$ particles. This difference can be explained by the fact that the $S t_{\eta} \leq 1$ particles are sensitive to the whole turbulence spectrum, whereas the heavier particles dampen small-scale motions due to their inertia.

According to our results (figures 2 and 3) $D$ does not universally indicate a characteristic cluster size of around $10 \eta$ [11] nor does the cluster size generally increase with $R e_{\lambda}$ [7]. Rather than a generally applicable size estimate, the maximum of $D$ indicates a length scale that is characteristic for a specific average particle number density. This does not contradict the experimental results (e.g. $[4,[5,7])$, but indicates that those results are only valid for a specific average particle number density.

Local concentrations show a more complex behaviour when gravity is introduced, as shown in the right column of figure 3. Gravity enhances preferential concentration at $S t_{\eta}>1$. The diminishing influence of the average particle number density on $D$ with increasing $S t_{\eta}$ is counterbalanced by this gravity effect (compare left and right column). For the highest numbers of particles there is a clear peak preferential concentration at $S t_{\eta}=1$ and a second, smaller peak at $S t_{\eta}=8$. For $N_{p}=16.5$ particles $/ \mathrm{m}^{3}$ the pattern is similar to simulations without gravity, with a peak at $S t_{\eta}=1$ and a decrease with higher Stokes numbers. For $N_{p}=16.5$ particles $/ \mathrm{m}^{3}$ there is an increase in $D$ at $S t_{\eta}=0.4$ to $S t_{\eta}=1$, but little change for higher Stokes numbers. Depending on average particle number density and gravity, it is possible to observe maximum preferential concentration at a peak other than $S t_{\eta} \approx 1$ (compare [6]) or even a near-independence of $S t_{\eta}$ (compare [7]).

The Reynolds number dependence of preferential concentration was investigated by comparing simulations for $S t_{\eta}=1$ at Reynolds numbers up to 
(a) $S t_{\eta}=0.4$, no gravity

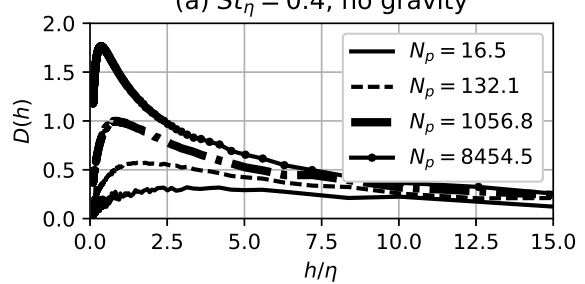

(b) $S t_{\eta}=1$, no gravity

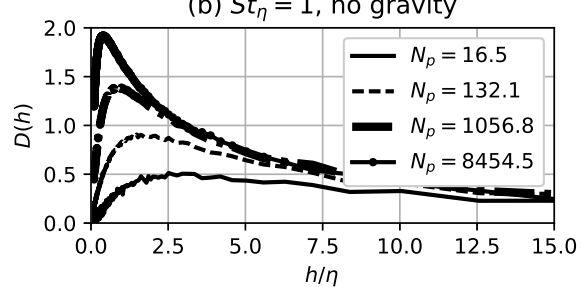

(c) $S t_{\eta}=2$, no gravity

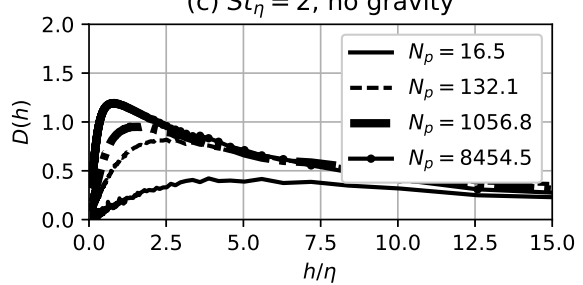

(d) $S t_{\eta}=4$, no gravity

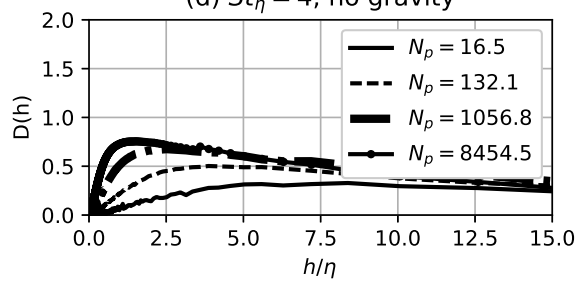

(e) $S t_{\eta}=8$, no gravity

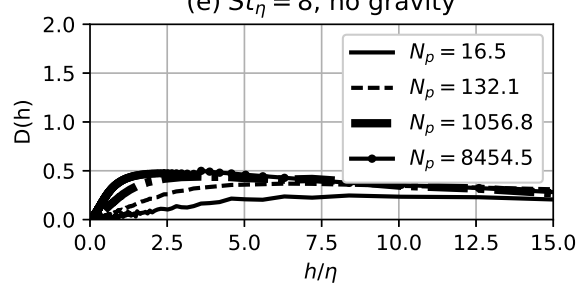

(f) $S t_{\eta}=0.4$, gravity

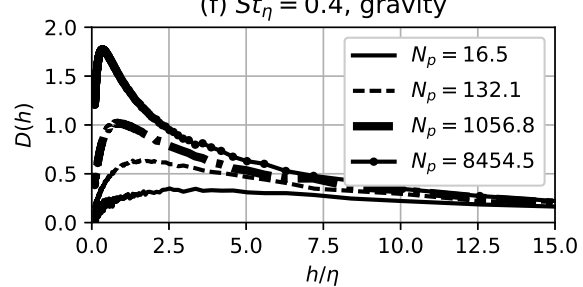

(g) $S t_{\eta}=1$, gravity

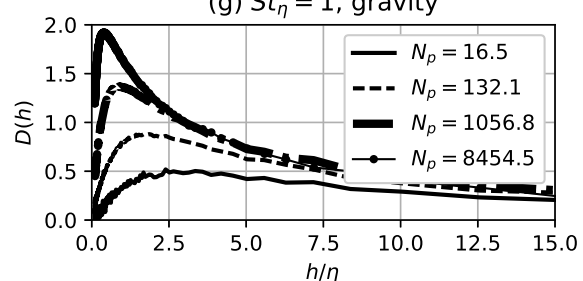

(h) $S t_{\eta}=2$, gravity

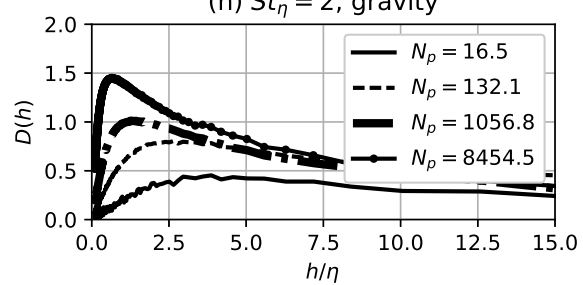

(i) $S t_{\eta}=4$, gravity
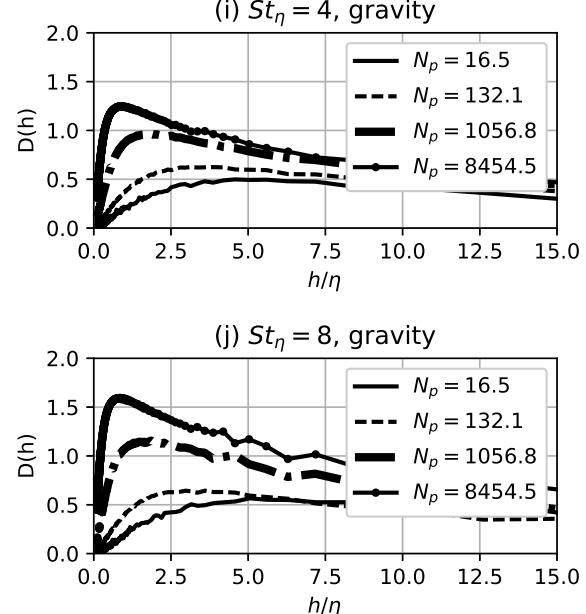

Figure 3: Stokes number and particle number density influence on $D$ at $R e_{\lambda}=45$. The left column shows results without gravity, the right column shows results including gravity effects. The Stokes numbers are (a)(f) $S t_{\eta}=0.4$, (b)(g) $S t_{\eta}=1$, (c)(h) $S t_{\eta}=2$, (d)(i) $S t_{\eta}=4$, (e)(j) $S t_{\eta}=8$. 
$R e_{\lambda} \approx 470$. The results for $R e_{\lambda} \approx 45, R e_{\lambda} \approx 250$ and $R e_{\lambda} \approx 470$ are shown in figure 4. The radial distribution function $g(r)$ shows a significant change between $R e_{\lambda} \approx 45$ and $R e_{\lambda} \approx 250$, but very similar values for the two highest Reynolds numbers. This is consistent with the results of other workers (e.g. Ireland et al. [2]) which suggest that $g(r)$ becomes independent of $R e_{\lambda}$ at sufficiently high Reynolds number. $D$ on the other hand shows a more complex behaviour when maintaining the average particle number density. The results for the Reynolds numbers investigated here show that the maximum of $D$ decreases with increasing $R e_{\lambda}$ (figure 4 (b)).

By varying the average particle number density it was found that a scaling behaviour with the Reynolds number similar to that exhibited by $g(r)$ can be ${ }_{125}$ achieved for $D$ when using the same number of particles per control volume. The $D$ measure for $(1 / 8)^{3}$ particles per control volume and $(1 / 4)^{3}$ particles per control volume is shown in figure 4. As for $g(r)$, there is a significant change between $R e_{\lambda} \approx 45$ and $R e_{\lambda} \approx 250$, but only a small change between $R e_{\lambda} \approx 250$ and $R e_{\lambda} \approx 470$ as shown in figures $4(\mathrm{c})$ and (d). Using the definition of the highest resolved wavenumber, it can be stated that $D$ becomes independent of the Reynolds number at sufficiently high Reynolds number if

$$
N_{p}^{(1 / 3)} \eta
$$

is kept constant, where $N_{p}$ is the number of particles per unit volume and $\eta$ is the Kolmogorov length. To our knowledge, such a scaling relation for $D$ is new. This relationship is simpler than the empirical relations established by Sumbekova et al. 7] for Voronoi diagrams.

Our results show that the strong non-linear effect of the global particle loading on local concentrations that has been observed in experiments [1, 7] can be reproduced by varying the number of particles in one-way coupled DNS. Our results cannot therefore be explained by the collective effects proposed by several authors [11, 7] in which regions with a high particle density collect further particles. This is because neither turbulence modulation by particles nor 
(a)

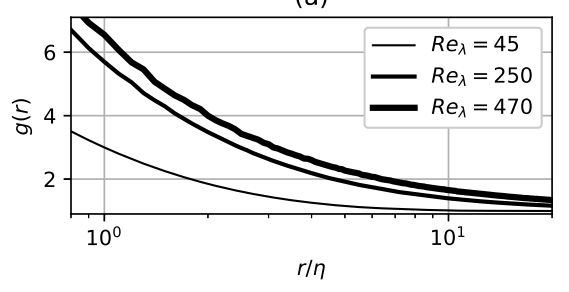

(c)

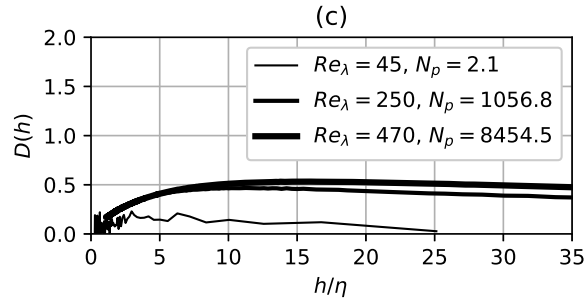

(b)

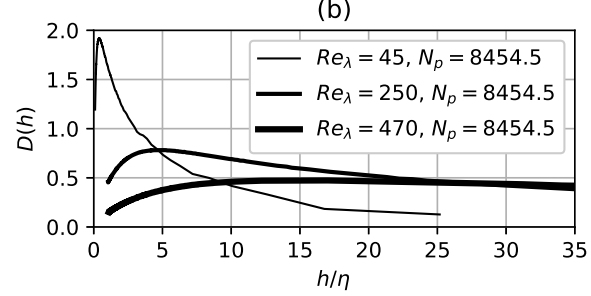

(d)

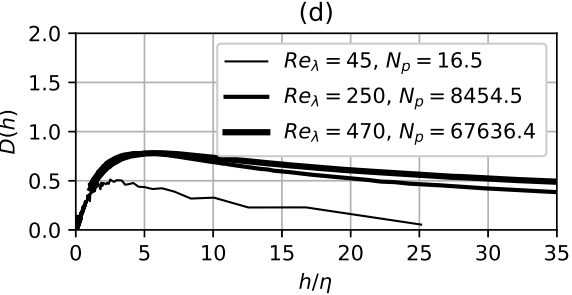

Figure 4: $R e_{\lambda}$ dependence of (a) $g(r)$ and (b) to (d) $D$. For $D$ the Reynolds number dependence is shown (b) for a constant average particle number density and (c),(d) for a constant number of particles per control volume.

particle-particle collisions which could lead to such effects are present in our simulations.

\section{Conclusions}

A non-linear dependence of local number concentrations in particle clusters, measured by $D$, has been found using one-way coupled direct numerical simulations in terms of average particle number density used in the simulations. The results explain observed differences reported in the literature between experimental and also computational results. The absence of turbulence modulation by particles and particle-particle collisions in the simulations means that these results cannot be explained by collective effects. It has been found that if the value of $N_{p}^{(1 / 3)} \eta$ is held constant, where $N_{p}$ is the number of particles per unit volume and $\eta$ is the Kolmogorov length, then the preferential concentration metric $D$ reaches a plateau at sufficiently high Reynolds number. Furthermore both cluster sizes and Stokes number dependence as measured by $D$ depend on 
the average particle number density.

\section{Acknowledgements}

The authors would like to thank Mr Tim Foat, Dr Steven Herring and Dr Simon Parker from the Defence Science and Technology Laboratory (DSTL) and Dr Zheng-Tong Xie from the University of Southampton for their very constructive and helpful feedback and suggestions.

This research is funded by the Defence Science and Technology Laboratory (DSTL), by EPSRC under Centre for Doctoral Training grant EP/L015382/1 and by EPSRC under the standard research grant EP/L000261/1 (UK Turbulence Consortium). We acknowledge the use of the IRIDIS High Performance Computing Facility, and associated support services at the University of Southampton, as well as the ARCHER UK National Supercomputing Service (http://www.archer.ac.uk) in the completion of this work.

\section{Data access}

The input parameters and fluid statistics of all model runs as well as the results shown in the figures are freely available from the University of Southampton repository at http://dx.doi.org/10.5258/SOTON/D0451. The particle restart files with particle positions and velocities can be provided by the authors on request.

\section{References}

[1] R. Monchaux, M. Bourgoin, A. Cartellier, Analyzing preferential concentration and clustering of inertial particles in turbulence, International Journal of Multiphase Flow 40 (2012) 1-18.

[2] P. J. Ireland, A. D. Bragg, L. R. Collins, The effect of Reynolds number on inertial particle dynamics in isotropic turbulence. Part 1. Simulations 
without gravitational effects, Journal of Fluid Mechanics 796 (2016) 617658.

[3] J. R. Fessler, J. D. Kulick, J. K. Eaton, Preferential Concentration of Heavy-Particles in a Turbulent Channel Flow, Physics of Fluids 6 (11) (1994) 3742-3749.

[4] Wood, A. M. and Hwang, W. and Eaton, J. K., Preferential concentration of particles in homogeneous and isotropic turbulence, International Journal of Multiphase Flow 31 (10-11) (2005) 1220-1230.

[5] A. Aliseda, A. Cartellier, F. Hainaux, J. C. Lasheras, Effect of preferential concentration on the settling velocity of heavy particles in homogeneous isotropic turbulence, Journal of Fluid Mechanics 468 (2002) 77-105.

[6] M. Obligado, T. Teitelbaum, A. Cartellier, P. Mininni, M. Bourgoin, Preferential concentration of heavy particles in turbulence, Journal of Turbulence 15 (5) (2014) 293-310.

[7] S. Sumbekova, A. Cartellier, A. Aliseda, M. Bourgoin, Preferential concentration of inertial sub-Kolmogorov particles: The roles of mass loading of particles, Stokes numbers, and Reynolds numbers, Physical Review Fluids 2 (2) (2017) 024302.

[8] L. R. Collins, A. Keswani, Reynolds number scaling of particle clustering in turbulent aerosols, New Journal of Physics 6.

[9] S. Sundaram, L. R. Collins, Collision statistics in an isotropic particleladen turbulent suspension .1. Direct numerical simulations, Journal of Fluid Mechanics 335 (1997) 75-109.

[10] S. J. Scott, A. U. Karnik, J. S. Shrimpton, On the quantification of preferential accumulation, International Journal of Heat and Fluid Flow 30 (4) (2009) 789-795. 
[11] R. Monchaux, M. Bourgoin, A. Cartellier, Preferential concentration of heavy particles: a Voronoi analysis, Physics of Fluids (1994-present) 22 (10) (2010) 103304.

[12] P. J. Ireland, A. D. Bragg, L. R. Collins, The effect of Reynolds number on inertial particle dynamics in isotropic turbulence. Part 2. Simulations with gravitational effects, Journal of Fluid Mechanics 796 (2016) 659-711.

[13] S. J. Scott, A PDF Based Method for Modelling Polysized Particle Laden Turbulent Flows Without Size Class Discretization, Ph.D. thesis, Imperial College London (2006).

[14] M. R. Maxey, J. J. Riley, Equation of motion for a small rigid sphere in a uniform flow., Phys. Fluids 26 (1983) 883-889.

[15] M. Frigo, S. G. Johnson, The design and implementation of FFTW3, in: Proceedings of the IEEE, Vol. 93, 2005, pp. 216-231.

[16] V. Eswaran, S. B. Pope, An Examination of Forcing in Direct Numerical Simulations of Turbulence, Computers \& Fluids 16 (3) (1988) 257-278.

[17] N. P. Sullivan, S. Mahalingam, R. M. Kerr, Deterministic forcing of homogeneous, isotropic turbulence, Physics of Fluids 6 (4) (1994) 1612-1614.

[18] J. C. Vassilicos, Dissipation in turbulent flows, Annual Review of Fluid Mechanics 47 (2015) 95-114.

[19] Y. Kaneda, T. Ishihara, M. Yokokawa, K. Itakura, A. Uno, Energy dissipation rate and energy spectrum in high resolution direct numerical simulations of turbulence in a periodic box, Physics of Fluids 15 (2) (2003) L21.

[20] P. Yeung, D. Donzis, K. Sreenivasan, Dissipation, enstrophy and pressure statistics in turbulence simulations at high Reynolds numbers, Journal of Fluid Mechanics 700 (2012) 5-15. 
[21] W. McComb, A. Berera, S. Yoffe, M. Linkmann, Energy transfer and dissipation in forced isotropic turbulence, Physical Review E 91 (4) (2015) 043013.

[22] A. U. Karnik, J. S. Shrimpton, Mitigation of preferential concentration of small inertial particles in stationary isotropic turbulence using electrical and gravitational body forces, Physics of Fluids 24 (7) (2012) 073301. 